\title{
Performance Comparison of Fractional Controllers and Artificial Intelligence Techniques
}

\author{
Mehmet Korkmaz and Omer Aydogdu
}

\begin{abstract}
Studying and applications of fractional PIDs (FO-PID) are getting popular for controlling the systems, properly. With this idea, it is aimed to unveil the advantages of FO-PID over traditional integer order PIDs (IO-PID). At the same time, in this paper, artificial intelligence techniques which are particle swarm optimization and genetic algorithm are used to determine which one have more effectiveness and to find system best parameters for both controllers. Results prove the supremacy of FO-PID controllers and PSO algorithm.
\end{abstract}

Index Terms-Fractional order controllers, particle swarm optimization, genetic algorithm, motor position control

\section{INTRODUCTION}

Fractional calculus is a generalization of traditional one which is suggested in the letter of Leibniz to L'Hopital, at the late-16th cc. with the assumption of "what if the degree of derivative is non-integer" [1]. Although the long term period until explored, it has not been found many areas to realize in the industry applications or academic studies [2], [3]. This is the due to the fact that, fractional calculus has heavy calculation on its base. Last two decades, however, it becomes widespread to utilize by favour of the computers and microprocessors which allow to make simpler estimating of fractional calculus. Analyzing the nature, most of the systems cannot fully identify with the integer derivative \& integral terms [4]-[7], on the score of the fact that this result obliges us to define the systems with fractional terms. In this point, fractional calculus is the very key for describing systems which have non-integer derivative and integral terms. During the recent years, it is seen that many systems are expressed with fractional terms. Not only systems representation but also controlling the system with fractional controllers is provides us with more freeness by virtue of extra derivative and integral order parameters.

Artificial intelligence (AI) techniques such as fuzzy systems, evolutionary and heuristic algorithms are mostly used obtaining the parameters in the control systems [8], [9]. They present effective conclusion for obtaining optimal points of problems by using numerical solution. One of which is genetic algorithm [10] has been found wide application field in the control engineering as a powerful tool searching optimal values. In addition to this type of algorithm, particle swarm optimization [8],[11] which is thought as one of heuristic algorithm has also suggested for the same

Manuscript received February 25, 2013; revised April 17, 2013. This work is supported by Selcuk University Scientific Research Fund, BAP, under the contact number 13701191 .

The authors are with department of electrical and electronics engineering, Selcuk University, Konya, PK 42075 Turkey (e-mail: mkorkmaz@ selcuk.edu.tr, oaydogdu@ selcuk.edu.tr). intention. PSO is inspired from the movement of flock of birds or school of fishes that similarly GA, optimizes problem by iteratively to obtain best candidates with regard to an accepted criteria. These influential algorithms have been applying many areas in engineering and academic research.

\section{FRACTIONAL SYSTEMS}

As it mentioned above, the terms integral \& derivatives are in the non-integer form for fractional calculus. It is obtained the unit feedback of the system (Fig. 1) considering in Laplace domain:

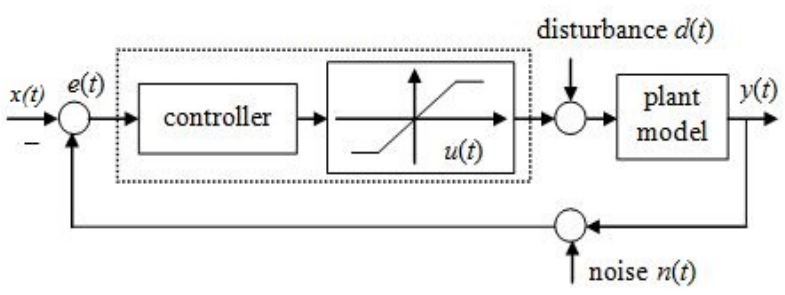

Fig. 1. Unit feedback system

$$
G(s)=\frac{Y(s)}{X(s)}=\frac{b_{m} s^{\beta m}+b_{m-1} s^{\beta m-1+\cdots+b_{0} s^{\beta_{0}}}}{a_{n} s^{\alpha_{n}+a_{n-1} s^{\alpha_{n-1+\cdots}}}}
$$

where; $\alpha_{n}>\alpha_{n-1}>\cdots \geq \alpha_{0}, \beta_{m}>\beta_{m-1}>\cdots \beta_{0} \geq$ $0, a_{k}(k=0,1,2, \ldots, n)$ and $b_{l}(l=0,1,2, \ldots, m)$

There are several definitions of fractional calculus realizing to applications in the literature. Grünwald-Letnikov and Riemann-Lioville definitions are the most used and well-known of its definitions. In the paper of Podlubny [12] it is suggested fractional PIDs by improving traditional ones utilizing from fractional calculus. According to the equation 2 it can be easily observed the forms of PIDs by changing the value of the $\lambda$ and $\mu$. For example, having the degree one, both $\lambda$ and $\mu$ gives the classical PID; if value of $\lambda$ is one and $\mu$ is zero gives the PI controller etc (Fig. 2). It is clear that taking the orders as a non-integer will make possible the system control more powerful and flexible in the system describing.

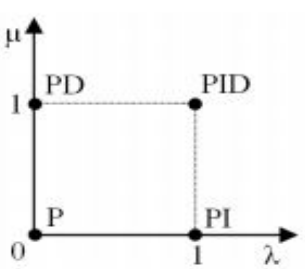

(a)

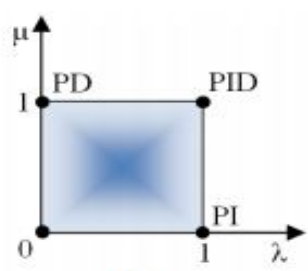

(b)
Fig. 2. Controllers in the $\lambda-\mu$ plain 


$$
G_{c}(s)=K_{p}+\frac{K_{i}}{s^{\lambda}}+K_{d} s^{\mu}=\frac{K_{d} s^{\lambda+\mu}+K_{p} s^{\lambda}+K_{i}}{s^{\lambda}}
$$

\section{Controller Design Strategies}

In the controller design, it grounds onartificial intelligence algorithms, genetic algorithm and particle swarm optimization.

Genetic algorithm (GA) is based on natural evolution that is the survival of the best individual in nature. The algorithm consists of three main phases which are mutation, selection and crossover. Randomly produced individuals are called the candidate solution represent the solution space. The progress starts from a solution set of randomly generated individuals and continue by calculating their fitness value which is desired point in the problem. Until reaching the stopping criteria or maximum iteration, it goes on the evolution that each iteration, individuals are operationalized specific processes which belong to GA (Fig. 3). Generally, individuals are represented as an array of bits; 0 and 1 . Real coded GA is, however, used in the applications to increase comprehensibleness.

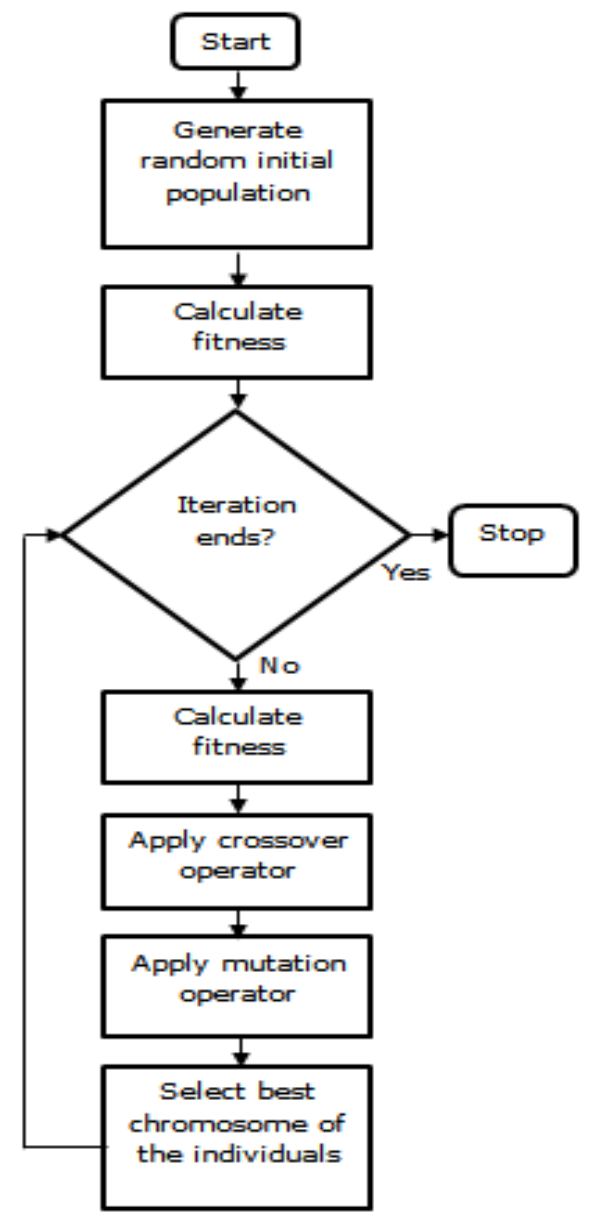

Fig. 3. Flowchart of Genetic Algorithm process

One of the other AI techniques, particle swarm optimization (PSO) is also very useful and effective algorithm for finding the best solution of optimization problems. With this purpose, PSO is applied to mentioned plant in the study as another optimization algorithm. PSO is originated from social behaviors ofanimals such as bird flocking.
According to algorithm, each individual's movements, called particle drift, are influenced by its local best position and also it is guided to of all best position in the solution space. For this reason, particle position and velocities are updated after each iteration regarding the its best value and equations $(3,4)$.

$$
\begin{gathered}
v_{i}^{k+1}=w \cdot v_{i}^{k}+c_{1} \cdot \text { rand }^{k} \cdot\left(\text { pbest }_{i}{ }^{k}-x_{i}{ }^{k}\right)+ \\
c_{2} \cdot \text { rand }^{k} \cdot\left(\text { gbest }_{i}{ }^{k}-x_{i}{ }^{k}\right) \\
x_{i}{ }^{k+1}=x_{i}{ }^{k}+v_{i}{ }^{k+1}
\end{gathered}
$$

$k \quad:$ iteration number

$w \quad$ : inertia weight

$c_{1}, c_{2} \quad$ : learning factors

rand : random number 0 to 1

$X_{i}^{k} \quad$ : particle variable

pbest $_{i}^{k} \quad$ : current best local position for each particle

gbest $_{i}^{k} \quad$ : current best global position for all particles

\section{Simulation AND EXPERIMENT RESUlts}

Simulation and experimental studies are applied for the system of Quanser SRV02 rotary module, motor position control (Fig. 4).

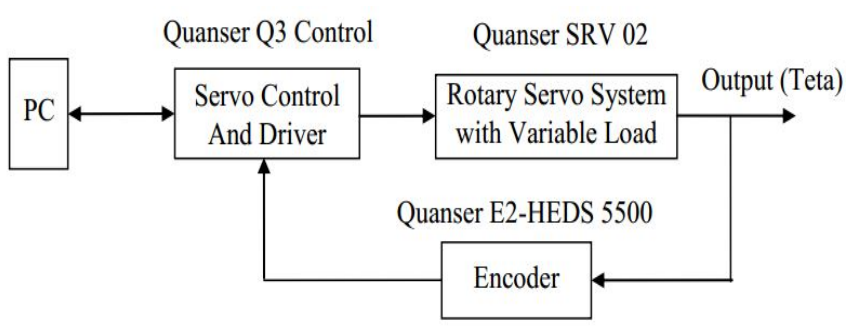

Fig. 4. Testing apparatus block scheme

Genetic algorithm and Particle swarm optimization techniques are used determining the parameters of controllers. First of all, it is determined mixed performance criteria (5) while finding best parameters of controllers for the mentioned plant. After that, simulations are executed for both algorithms 50 times and solution space checked during the new individuals (for GA) or velocity-positions (for PSO) if any unstable points. In this case any undesired points are dismissed from solutions.

$$
J=\int_{0}^{t_{s i m}}\left(w_{1}|e(t)|+w_{2} u^{2}(t)\right) d t
$$

Here $w_{1}$ and $w_{2}$ weighting coefficients and values are 0.999 and 0.001 , respectively.

$K_{\mathrm{p}}$ proportional gain, $K_{\mathrm{i}}$ integral gain, $K_{\mathrm{d}}$ derivative gain, $\lambda$ integral order and $\mu$ derivative order are limited for fair comparison of techniques and controllers as accepting the boundaries follows:

$$
K_{p}=[0,2] ; K_{I}=[0,2] ; K_{d}=[0,1] ; \lambda=[0,1] ; \mu=[0,1] \text {. }
$$

To begin with Fig. 5 that it shows the IO-PID controller design with different kinds of algorithms, GA \& PSO. 
Subsequent curves, Fig. 6 shows the IO-PID controller effects that extra load is added the system at the beginning to observe controller effects to change in the load disturbance. Similarly, Fig. 7 and 8 is worked for the same reason with a different controller, FO-PID. These four outputs reveal the superiority of PSO algorithm over GA for both controller designs.

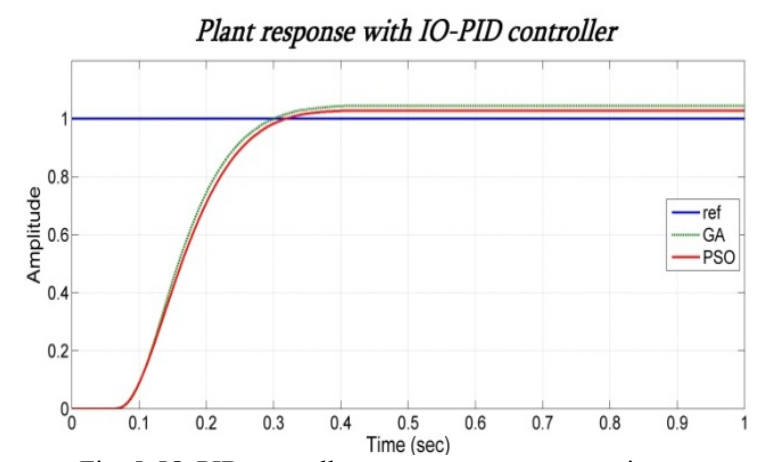

Fig. 5. IO-PID controller output response to step input

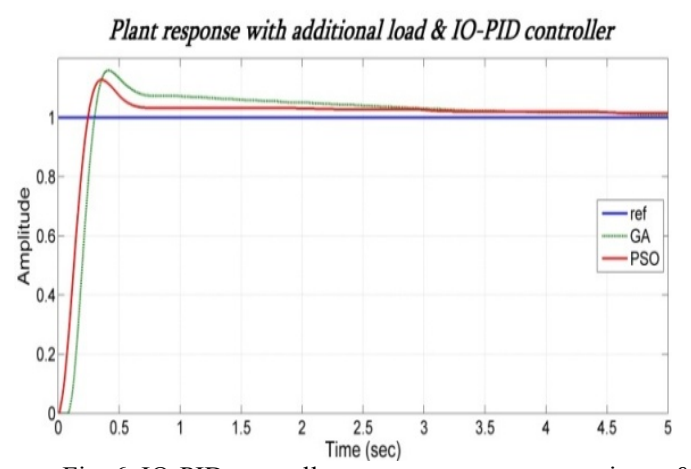

Fig. 6. IO-PID controller output response to step input \& extra load

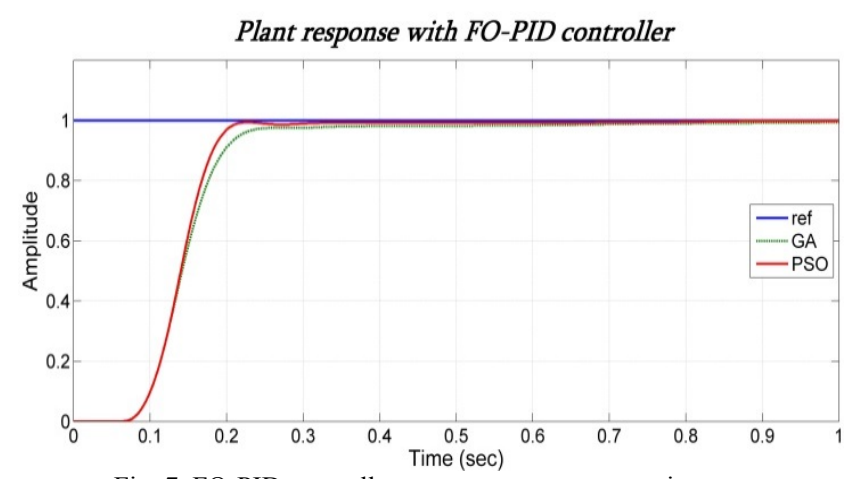

Fig. 7. FO-PID controller output response to step input

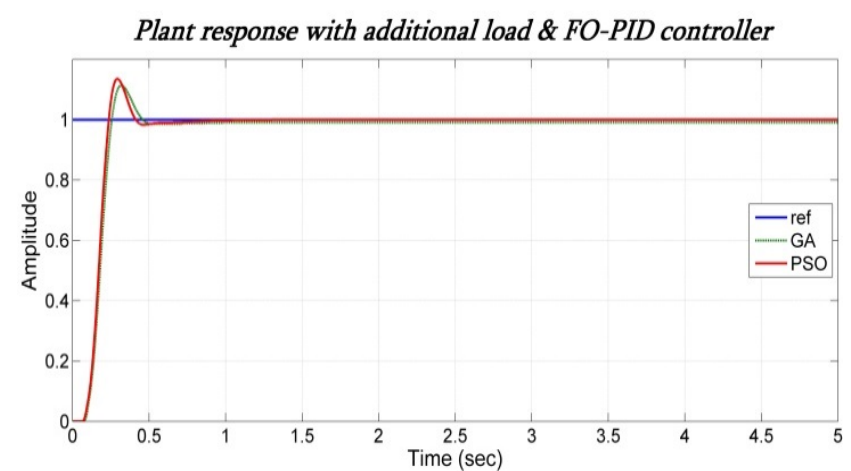

Fig. 8. FO-PID controller output response to step input \& extra load

On the other hand, next four Fig. (9, 10, 11 and 12) goal are to bring forward the impact of the FO-PID controller. For instance, looking into the Fig. 9 both IO-PID and FO-PID controllers have been designed by using GA technique that algorithm are executed 50 times, mutation and crossover ratios are chosen as a value of 0.5 . At the same thought, experiments are repeated by adding accessional load in order to observe additional load effect to system for both controllers (Fig. 10). After experiments it has been seen the advantages of the FO-PID controller. Hereunder, even though the designs are realized with several techniques the result which is superiority of FO-PID is the same. Beside the system have robustness the load disturbance.

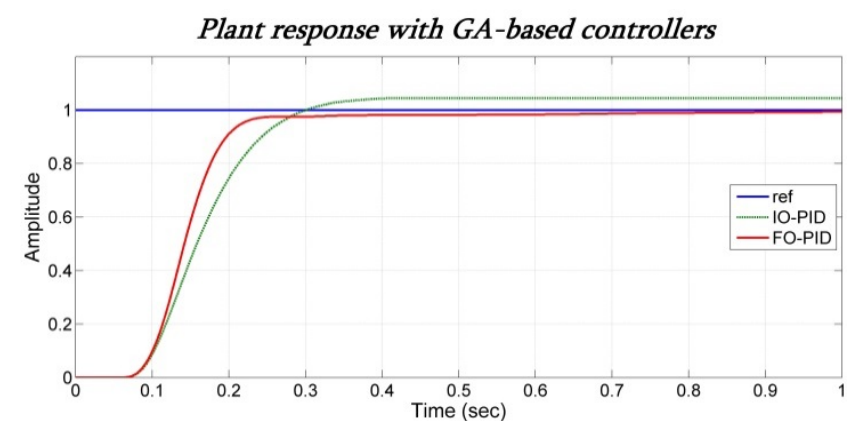

Fig. 9. GA-based controller output response to step input

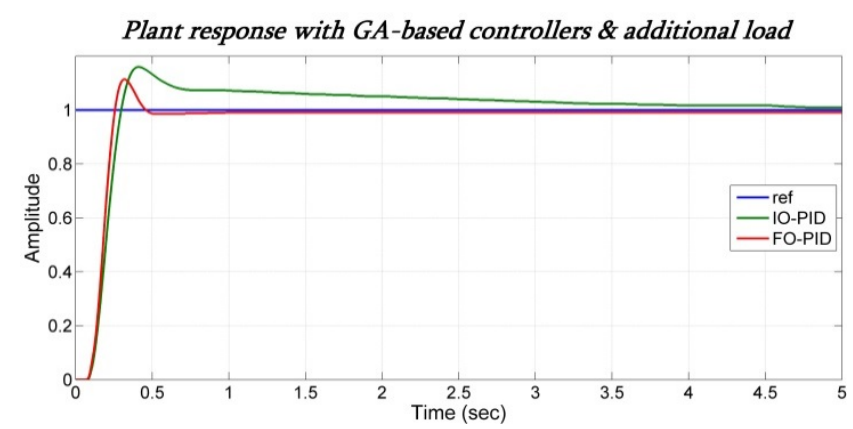

Fig. 10. GA-based controller output response to step input \& extra load

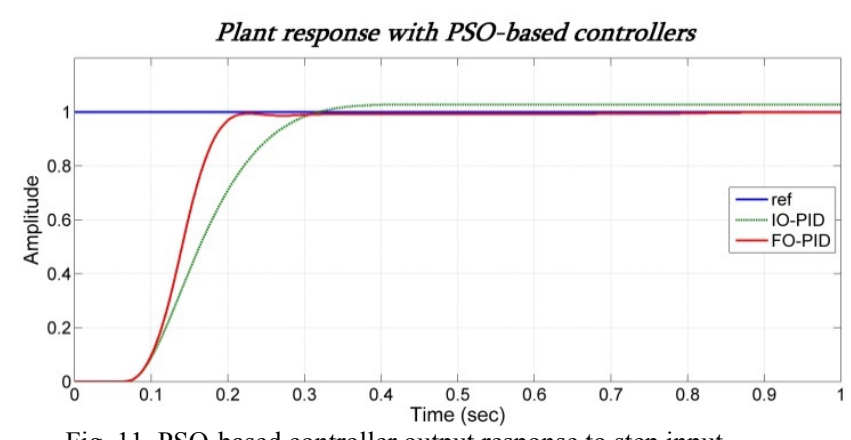

Fig. 11. PSO-based controller output response to step input

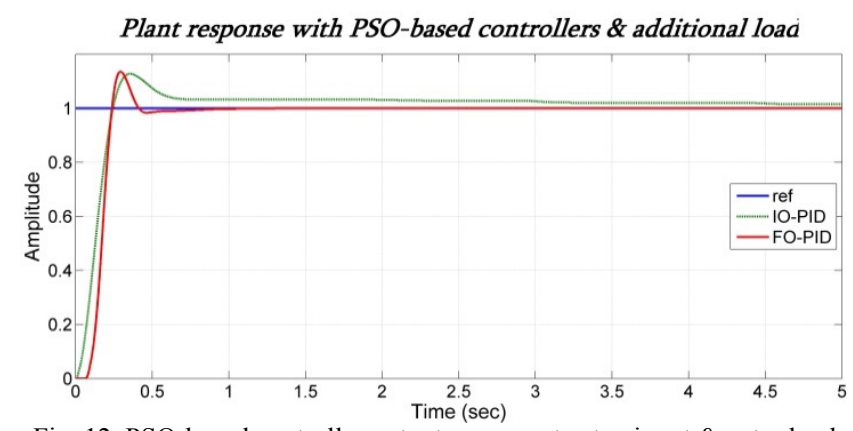

Fig. 12. PSO-based controller output response to step input \& extra load

\section{CONCLUSIONS}

In this paper, it is searched the effectiveness of the artificial intelligence techniques over the fractional and 
integer PID controllers. While obtaining the results, it is benefit from Quanser SRV02 Rotary Servo Module, position control experiment. First of all, system's best controller parameters are found by using simulations with the mentioned algorithms. Later the parameters found are applied the testing apparatus, position control experiment. On the other hand, it is desired to prove the robustness of the FO-PID controllers against to the load variations. For this purpose, experiments are firstly executed with the initial parameter conditions next, extra load is added to system. According to the study, three main results are obtained that first of all FO-PID structure has superiority over IO-PID controller; secondly FO-PID controller is the robust to the load disturbance. Lastly, PSO algorithm are better and faster than Genetic Algorithm to get optimum parameters of problems and.

\section{REFERENCES}

[1] K. B. Oldham and J. Spanier, The fractional calculus, New York: Academic, 1974.

[2] F. Padula and A. Visioli, "Tuning rules for optimal PID and fractional-order PID controllers," Journal of Process Control, vol. 21 no. 1, pp. $69-81,2011$.

[3] Y. Luo, Y. Q. Chen, C. Y. Wang, and Y. G. Pi, "Tuning fractional order proportional integral controllers for fractional order system," Journal of Process Control, vol. 20, no. 7, pp. 823-831, 2010.

[4] C. H. Lee and F. K. Chang, "Fractional-order PID controller optimization via improved electromagnetism-like algorithm," Expert Systems with Applications, vol. 37, no. 12, pp. 8871 - 8878, 2010.

[5] M. Caputo, Elasticita e dissipacione, Bologna: Zanichelli, 1969.

[6] Y. Chen, D. Xue, and H. Dou, "Fractional calculus and biomimetic control," in Proc. IEEE int. conf. on robotics and biomimetics, pp. 347 , 2004.

[7] T. F. Nonnenmacher and W. G. Glockle, "A fractional model for mechanicalstress relaxation," Philosophical Magazine Letters, vol. 64, no. 2, pp. 89-93, 1991.

[8] M. Zamani, K. G. Masoud, N. Sadati, and M. Parniani, “2009 Design of a fractional order PID controller for an AVR using particle swarm optimization," Control Engineering Practice, vol. 17, no. 12, pp. 1380 $-1387$.

[9] C. H. Lee and F. K. Chang, "Fractional-order PID controller optimization via improved electromagnetism-like algorithm," Expert Systems with Applications, vol. 37, no. 12, pp. 8871 - 8878, 2010.

[10] D. E. Goldberg, Genetic Algorithms in Search, Optimization and Machine Learning. Reading, MA Addison-Wesley, 1989.

[11] J. Kennedy and R. Eberhart, "Particle swarm optimization," in Proc. of EEE International Conference on Neural Networks, vol. 4, pp. 1942-1947, 1995.

[12] I. Podlubny, "Fractional-order systems and PI $\lambda \mathrm{D} \mu$ controllers," Automatic Control, IEEE Transactions, vol. 44, no. 1, pp.208-21, 1999.

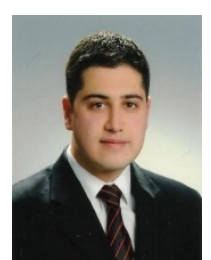

Mehmet Korkmaz received the B.Sc. degree from Department of Electrical and Electronics Engineering, Selcuk University, ,Konya, Turkey, in 2009 and still continues of his master studies in the system control area from 2011 at the same department and university.

Korkmaz graduated in 2009 then worked about 2 years in such different industrial areas. After his work experience he has started to work first at Aksaray University then at Selcuk University as a researcher since 2011. Korkmaz has publications in the fractional controller field that still resume the studies in the same focus.

Mr. Korkmaz graduated from B.Sc. taking the third degree within of all students. He has been working as a research assistant at Selcuk University since 2011 .

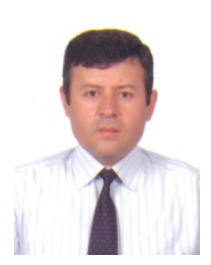

Omer Aydogdu received the B.Sc. and M.S. degrees from Department of Electrical and Electronics Engineering, Selcuk University, Konya, Turkey, in 1995 and 1999 respectively. Ph.D. degree from the Department of Electrical and Electronics Engineering and still continues of his studies area from 2006 at the same department and university.

Aydogdu have graduated in 1995 later he has started to work at Selcuk University since 1996. Aydogdu have graduated in 1995 later he has started to work at Selcuk University since 1996. Aydogdu has many publications in the different areas of control engineering that he still maintains the studies in the same focus. 